FORMATION Formation emploi

Revue française de sciences sociales

114 | Avril-juin 2011

Pêle-mêle

\title{
L'intermédiation à la croisée des logiques d'acteurs : Les psychologues du travail
}

Intermediation at the intersection of actors' approaches: occupational psychologists

Intermediation am Scheideweg der Logiken der Akteure: Arbeitspsychologen

La intermediación en el cruce de las lógicas de actores : los psicólogos del trabajo

\section{Benjamin Saccomanno}

\section{OpenEdition}

Journals

Édition électronique

URL : http://journals.openedition.org/formationemploi/3347

DOI : 10.4000/formationemploi.3347

ISSN : 2107-0946

Éditeur

La Documentation française

Édition imprimée

Date de publication : 31 juillet 2011

Pagination : $39-54$

ISSN : 0759-6340

\section{Référence électronique}

Benjamin Saccomanno, «L'intermédiation à la croisée des logiques d'acteurs : Les psychologues du travail », Formation emploi [En ligne], 114 | Avril-juin 2011, mis en ligne le 01 août 2013, consulté le 30 octobre 2020. URL : http://journals.openedition.org/formationemploi/3347 ; DOI : https://doi.org/ 10.4000/formationemploi.3347 


\section{Profession}

\section{L'Intermédiation à la croisée des logiques d'acteurs: les psychologues du travail}

Benjamin Saccomanno*

\section{En recherchant une solution personnalisée pour des demandeurs d'emploi, le dispositif d'appui à l'élaboration de projets de formation apparaît comme une co-construction qui intègre non seulement des enjeux relatifs au bénéficiaire, à l'organisation, au marché du travail mais aussi à la position professionnelle du psychologue.}

Les positions d'opérateurs de l'action publique auprès de bénéficiaires représentent un terrain d'analyse qui condense de nombreuses problématiques. Intermédiaire, leur activité s'inscrit, d'un côté, dans des enjeux macro-sociaux liés aux politiques dont ils assurent la mise en œuvre et, de l'autre, dans des configurations singulières portées par les destinataires auprès de qui ils travaillent. Cette activité est ainsi prise en tension entre différentes logiques pouvant en orienter l'issue. À partir du cas des psychologues du travail officiant au sein du service public de l'emploi (SPE), cet article étudie l'issue d'un dispositif d'accompagnement de projets individuels de formation comme une co-construction entre différentes logiques ajustées entre elles par le psychologue.

Comprises comme des systèmes de représentations et de valeurs qui vont orienter l'action vers un but précis (Nizet, 2009), les logiques définissent des points de vue, des catégories de jugement et des registres de qualification spécifiques de l'action. Par sa position et ses fonctions, le psychologue se trouve à un croisement de logiques spécifiques qu'il convient alors de coordonner. Il est ainsi à l'intersection de contraintes organisationnelles et politiques, produites notamment par les réorganisations des services (Weller, 1999; Dubar, 1989, Dubois, 2001). Il met ensuite en œuvre des programmes d'action qui révèlent des enjeux différenciés eu égard à une pluralité de destinataires (Bessy et alii, 1995). Son travail quotidien le conduit, d'une part, à utiliser des outils et dispositifs porteurs

* Benjamin Saccomanno est doctorant en sociologie au Centre d'études et de recherche Travail Organisation Pouvoir de l'université Toulouse le Mirail. Sa thèse porte sur le travail de co-construction des projets et des ambitions chez des adultes en formation professionnelle à I'AFPA (Association nationale pour la formation professionnelle pour adultes). 


\section{Encadré 1}

\section{L'économie des conventions : la coordination de l'action par les jugements}

Initié à la fin des années 80 , le programme de l'économie des conventions articule les rationalités et modalités de coordination de l'action avec les valeurs et conceptions des acteurs. Ces dernières ne sauraient être réduites à de simples préférences: en soutenant un partage de conventions quant à la perception et l'évaluation des situations, elles participent pleinement à la coordination d'acteurs ainsi accordés dans leurs conceptions du bien commun (c'est-à-dire une justification d'ordre général pouvant s'appliquer ensuite aux multiples configurations locales concernées). Cet accord est donc un enjeu fort en présence d'une pluralité de conventions ; son établissement devient alors le point d'entrée des recherches se référant à ce programme.

Le sens du bien commun s'exprime notamment lors de situations d'épreuve (comme une procédure de sélection par exemple) qui mobilisent des principes d'action et de jugement tels que la justice, et que les acteurs et organisations appliqueront selon des registres qui rendront compte d'effets de contexte et de position des acteurs. Ces registres s'expriment par le biais de règles, d'instruments, de supports mais aussi d'éléments de langage. Largement alimenté depuis par des travaux empiriques et théoriques, tant en économie qu'en sociologie, ce cadre d'analyse renouvelle le regard sur les activités et situations dans lesquelles des jugements de valeur et des considérations normatives interviennent dans le cours de l'action et de l'échange. Les rencontres entre offre et demande de travail fournissent logiquement des terrains particulièrement propices à ces approches.

Voir notamment: "L'économie des conventions " (numéro spécial de la Revue économique, sous la dir. de F. Eymard-Duvernay, mars 1989); "Justesse et justice dans le travail " (n 33 des Cahiers du Centre d'études de l'emploi, sous la dir. de L. Boltanski et L. Thévenot, 1989) ; L'économie des conventions, méthodes et résultats. Tome 1 Débats, tome 2 Développements (La Découverte, 2006); Au risque de l'évaluation. Salariés et candidats à l'emploi soumis aux aléas du jugement (sous la dir. de M.-C. Bureau, E. Marchal, Presses universitaires du Septentrion, 2005).

des rationalités de leurs producteurs (Boussard, 2001 ; Boussard, Maugeri, 2003) ; d'autre part, des données relevant de la subjectivité des bénéficiaires que les métiers relationnels font émerger (Divay, 2008). Enfin, sa propre subjectivité de psychologue du travail s'exprime dans son genre professionnel; celui-ci est compris comme un corps intermédiaire symbolique d'appréciation des situations de travail et qui exprime le rattachement à la fonction et non à l'organisation (Clot, 1999). La préconisation institutionnelle résultera donc de l'agencement entre ces logiques agissant sur le déroulement du programme étudié.

Tout d'abord, des repères objectifs encadrent l'action du psychologue du travail. Ils définissent des normes d'appréciation et de jugement de la situation. Ils font partie du cadre de l'interaction; leur présence et leur implication marqueront la préconisation finale de l'empreinte organisationnelle dans la mesure où celle-ci devra questionner le sens qu'ils confèrent à une « juste » décision. Nous aborderons ce point dans un premier temps de l'article. Mais nous verrons ensuite que des éléments variables agissent ici, allant d'un niveau global, comme la situation du marché de l'emploi, à un niveau local, avec les problématiques du bénéficiaire; ils configurent aussi l'interaction et ses enjeux. Dès lors, il revient au psychologue d'intégrer ces différents niveaux d'enjeux dans sa préconisation; il s'agit d'articuler des principes de justice et de justesse, articulation nécessaire à l'évaluation lors de la sélection de candidatures à des formations (Eymard-Duvernay, 2008). L'analyse des activités concrètes du psychologue révèle comment les différentes logiques en présence font du travail du psychologue l'enjeu de leur expression. Nous nous intéresserons particulièrement aux écarts qui existent entre les enjeux de ces logiques. Nous noterons que l'ajustement en vue d'une acceptabilité de la candidature ne renvoie pas nécessairement à des éléments relatifs à la démarche du bénéficiaire. Le travail des 


\section{Encadré 2}

\section{Les psychologues du travail dans le service public de l'emploi (SPE)}

En France, officient environ 40000 psychologues du travail, titre certifié par l'obtention d'un diplôme (master 2) et d'une durée minimum de stages pratiques. Au moment de notre enquête, l'AFPA (Association nationale pour la formation professionnelle des adultes) faisait partie des principaux employeurs de la profession (700 au niveau national). Avant leur rattachement au Pôle emploi, ces psychologues relevaient de services d'orientation professionnelle (SOP : en général un par département, voire plus selon la taille des territoires), eux-mêmes coordonnés par les Centres régionaux d'orientation professionnelle (CROP).

En Midi-Pyrénées, 40 psychologues sont recensés au sein de l'AFPA. Au niveau des spécialisations professionnelles, nous relevions une répartition de l'ordre de 60/40 entre les psychologues formés aux problématiques du travail et ceux de formation clinicienne. La profession est fortement féminisée avec environ $88 \%$ de femmes parmi les psychologues cliniques contre $78 \%$ parmi les psychologues du travail (Le Bianic, 2005, p. 341 ).

En 2006, 81 \% des stagiaires de l'AFPA ont préalablement été reçus par ces psychologues. La loi $n^{\circ} 2008-126$ du 13 février 2008, l'avis du conseil de la concurrence n 08-A-10 du 18 juin 2008 et enfin la loi n⿳2 2009-1437 du 24 novembre 2009, ont organisé le transfert des missions d'orientation vers le Pôle emploi, au plus tard le 1er avril 2010 : 75 \% des psychologues de l'AFPA furent concernés. Outre l'objectif de rationalisation des services publics, ce transfert a été motivé par une suspicion de favoritisme de la part des psychologues envers le dispositif AFPA qu'ils alimenteraient en priorité, alors que dans le même temps le marché de la formation professionnelle s'ouvre à la concurrence.

Outre l'activité d'orientation et d'accompagnement des projets de formation, les services de psychologues de l'AFPA ont à charge le suivi psychopédagogique des stagiaires ainsi que des activités d'études, de bilan GPEC (gestion prévisionnelle des emplois et des compétences) et de conseil en entreprise (le marché privé représente $15 \%$ de l'activité).

\section{Encadré 3 \\ La méthode}

Menée en 2008-2009, en Midi-Pyrénées, dans le cadre d'un doctorat de sociologie, l'enquête s'est déroulée alors que le transfert des psychologues vers le Pôle emploi était annoncé mais non acté. Nous nous appuyons principalement sur des données documentaires et qualitatives : 15 entretiens semi-directifs d' 1 h 30 en moyenne ont été menés avec des psychologues encore rattachés à l'AFPA (Association nationale pour la formation professionnelle des adultes). Nous avons fait varier en premier lieu les types d'approches (clinicienne/du travail) et l'ancienneté dans le service (de 18 à 3 ans), afin de saisir de potentielles différences dans les pratiques professionnelles et dans les représentations générales de la place du psychologue au sein du service public de l'emploi. Ceci fut complété par l'observation de passations de tests et de rendez-vous (16).

Afin de relativiser les discours (organisés par un guide d'entretien interrogeant l'accompagnement, l'orientation, la mesure des compétences, les coopérations avec d'autres services, institutions ou avec l'encadrement), nous intégrons des données tirées d'entretiens avec d'autres acteurs tels que des formateurs (1 1), des ingénieurs de formation et d'orientation (6), des conseillers Pôle emploi (14) ou encore des membres de l'encadrement national et régional de l'AFPA (9), ainsi que des stagiaires (45) avec lesquels nous sommes entre autres revenus sur leur recrutement. 
psychologues étudiés révèle des évolutions organisationnelles du SPE venant structurer l'espace dédié à l'appui subjectif et relationnel et donc, à terme, les contenus des projets de formation. Organisée autour du concept de sécurisation des parcours professionnels à partir d'un travail sur l'employabilité des individus (Merle, 2009 ; Gazier, 2009), l'action du SPE reconfigurerait ainsi la conception du travail des psychologues.

\section{NORMALISER LE JUGEMENT AUTOUR DES LOGIQUES D'EFFICACITÉ}

«Plus question de se former pour une promotion, ni seulement pour acquérir une qualification, un diplôme, il s'agit de maintenir son employabilité pour conserver son emploi ou tenter d'en (re)trouver un " analysaient Dubar et Gadéa en 1998, soutenant l'idée d'un « envahissement du champ de la formation par l'économique ». De ce point de vue, les restructurations et législations opérées depuis dans le champ n'ont fait que confirmer leur analyse (Mériaux, 2009). Le dispositif considéré dans cet article - le « S2 »-doit alors être compris dans cette conception du système de formation. Son élaboration, son déroulement et les outils requis révèlent une fonction d'encadrement de l'activité du psychologue qui le met en œuvre.

Le S2 consiste en une prestation de conseil et d'accompagnement dont l'objet est de co-construire et de valider, avec le bénéficiaire, un projet de formation comme la « solution la plus adaptée à la personne», nous indique un responsable des services d'orientation. Afin d'analyser comment s'élabore cette solution, il convient d'insérer le dispositif dans son environnement organisationnel (Boussard et alii, 2010 , pp. 157-173). Sa mise en œuvre fait ainsi intervenir d'autres acteurs du réseau constitué par le SPE autour du système de formation. Leurs actions contribuent à normaliser le déroulement du processus. Pour cela, ils encadrent l'interaction avec le bénéficiaire, lors des activités de contractualisation, de mesure des capacités, puis durant l'évaluation de cette interaction. Leurs apports fournissent des supports de jugement des projets et des profils des bénéficiaires. Cela permet la coordination des membres du réseau d'action autour de conventions communes (Eymard-Duvernay, 1989) et d'une économie du codage, celle-ci proposant une mise en forme stabilisée et normalisée des critères d'évaluation (Thévenot, 1983). Cette première partie sera donc consacrée à la façon dont le cadre et les ressources tendent à normaliser les jugements opérés par le psychologue. Nous nous intéresserons particulièrement à ces supports de cadrage que sont le contrat, l'évaluation, les guides et référentiels d'activité et enfin les outils de mesure des capacités.

\section{Cadrage préalable : le contrat et l'évaluation des services}

Le premier élément organisationnel qui inaugure l'interaction est la contractualisation passée entre le SPE et l'administré. Elle qualifie ce dernier en tant que bénéficiaire de la prestation. Depuis les années 90, la pratique de la contractualisation s'est particulièrement développée dans le secteur social de l'action publique. Elle a vocation à susciter des formes d'autonomie, d'implication et de responsabilisation chez le signataire (Pattaroni, 2007). Elle s'inscrit ainsi dans le cadre des politiques d'activation (Beyraud, Eydoux, 2009). En effet, son destinataire est mobilisé en tant qu'acteur de l'élaboration de son accompagnement, ouvrant la voie à la co-construction des décisions. Le contrat organise l'interaction en posant une obligation d'engagement mutuel entre les parties concernées qui travailleront à un rapprochement autour d'intentions conjointes (Loubat, 2000). Cet échange est donc encadré par des règles, des objectifs et des modalités d'action : le bénéficiaire soumet sa démarche à l'épreuve du jugement par le dispositif ; une part de la responsabilité lui est transférée quant à l'issue du S2, sa signature induisant l'acceptation de la préconisation finale. Du côté du psychologue, ce contrat se superpose à ce qui fonde une part de son rôle au sein du SPE, c'est-à-dire une activité d'information, d'évaluation et d'orientation des projets de formation.

À l'autre borne du S2, l'organisation du processus induit une nouvelle phase de cadrage : l'évaluation de la performance du dispositif par son financeur, la DGEFP. Axe du nouveau management public (Lagroye, 2002), l'évaluation correspond à un gouvernement de l'action par les objectifs; les objectifs, 
critères et indicateurs d'appréciation sont établis en amont. La coordination de l'action publique repose elle-même sur des contractualisations entre services ; les contrats de progrès qui délèguent la mission de formation de l'État vers l'AFPA fixant les attentes du commanditaire sous forme de seuils ou valeurs à

\section{Encadré 4}

\section{Le service d'appui à la définition d'un projet de qualification (S2)}

Mis en place au sein du service public de l'emploi (SPE) en 1999, le "S2 » (service d'appui à la construction d'un parcours de formation) est une prestation dont la durée varie entre 3 et 5 heures. Il est activé lorsque le conseiller emploi établit un besoin de formation ou que l'administré fait reconnaître un besoin de formation.

Juridiquement, il est intégré au contrat de progrès reliant l'opérateur de formation à l'État par le biais de la DGEFP (Délégation générale à l'emploi et à la formation professionnelle), concrétisant « la volonté des pouvoirs publics de mieux coordonner les compétences respectives des institutions afin de lutter contre le chômage ». La DGEFP passe commande auprès du service des psychologues afin de recevoir un nombre pré-établi de demandeurs d'emploi ayant formulé un besoin de formation auprès de leur conseiller ANPE (Agence nationale pour l'emploi) / Pôle emploi. Le conseiller emploi prend donc un rendez-vous sur l'agenda du service d'orientation professionnelle (SOP) et adresse ainsi quasi automatiquement la personne. Au moment de notre enquête, le S2 représente, à l'échelon national, 75 \% de l'activité des Centres régionaux d'orientation professionnelle (CROP).

En 2008, au niveau national, 257560 personnes ont été accueillies pour un service d'appui à la construction d'un parcours de formation, dont 200316 (environ $78 \%$ ) adressées par un conseiller emploi. Parmi elles, $65 \%$ des demandeurs d'emploi se sont engagés dans un programme qualifiant (AFPA ou autre organisme); $28 \%$ sont dirigés vers le marché de l'emploi ou bien Pôle emploi pour retravailler leur projet professionnel ; 3 \% ont abandonné la prestation en cours ; 3,6 \% l'ont terminée l'année suivante.

Pour 2008, la répartition par âge des bénéficiaires de $\$ 2$ est la suivante : moins de 25 ans : $26,8 \%$; $25-35$ ans : $34 \% ; 35-45$ ans : $24,7 \% ; 45$ ans et plus : $14,5 \%$. Les projets de formation apparaissent, pour une bonne part d'entre eux, comme un réajustement opéré au cours du premier tiers de la trajectoire professionnelle. Ce sont majoritairement des hommes (61,4\%) et, parmi les demandeurs d'emploi, 65,5 \% sont au chômage depuis moins d'un an. Les projets s'initient souvent durant les premiers temps de l'indemnisation, en raison notamment de la durée limitée des droits ouverts, mais aussi en lien avec les politiques de l'emploi visant à replacer rapidement les demandeurs dans des postures actives.

Enfin, les stagiaires de l'AFPA (Association nationale pour la formation professionnelle des adultes) en formation de niveau $\mathrm{V}$ (BEP/CAP) et $\mathrm{V}$ bis ( 1 année dans le secondaire) représentent plus de $70 \%$ des stagiaires de 2008. 17,2 \% des stagiaires visent des titres de niveau IV (Bac) et 5,9\% au-delà (Niveaux III et II : Bac $+2, \mathrm{Bac}+3-4)$.

Les effets de ce service se font sentir sur le taux de validation des parcours $179 \%$ contre $61 \%$ n'en ayant pas bénéficié, source : INOIP - Institut national de l'orientation et de l'insertion professionnelles) et sur le taux d'accès à l'emploi post formation (71 \% contre $66 \%$ ).

Sources : AFPA, Rapport d'activité 2008 ; Annexe Formation au projet de loi des finances, 2010 ; Brochure AFPA, DEAT, INOIP « Services d'orientation 2004 ». 
atteindre'. En couplant l'action évaluée aux objectifs visés en aval, l'évaluation permet de prescrire et de contrôler les procédures. À cet effet, elle s'appuie sur un travail continu de codification, saisie et transmission informatique des données relatives aux prestations et actes professionnels effectués, de la prise de rendez-vous à la passation de tests psychotechniques (Le Bianic, Rot, 2009). La prévision et le suivi financier des actions menées s'inscrivent dans la logique de justification comptable de la LOLF².

De l'entrée à la clôture de la prestation, l'activité du psychologue se déroule au sein d'un processus organisationnel codifié, prescriptif et fixant les règles de son déroulement et de sa propre appréciation. Les conceptions de l'efficacité, établies et valorisées par ces protocoles formalisés, ont alors pour effet de rabattre l'action des professionnels sur les logiques institutionnelles structurant le processus. De même, les supports de jugement utilisés durant l'interaction - tels que les outils d'appréciation des compétences - contribuent à objectiver les attentes institutionnelles en stabilisant les critères d'évaluation des projets.

\section{Les outils de positionnement: rationaliser les pratiques et normaliser les jugements}

Le S2 interroge la pertinence d'un projet de formation. Pour cela, le psychologue va évaluer la concordance potentielle entre le profil du bénéficiaire et les prérequis de la formation. Il dispose de ressources dont les usages et contenus vont l'aider dans son jugement tout en rationalisant le déroulement de l'interaction.

\footnotetext{
${ }^{1}$ Pour la période concernant notre enquête, nous trouvons : Fixation d'objectifs pour les préconisations : le taux de renvoi sans solution de formation doit être inférieur à $25 \%$ (Cette issue ne donne pas lieu à une rétribution financière prévue dans le contrat d'objectifs passé avec la DGEFP) ; Cadrage temporel de l'action : la durée entre l'adressage par le Pôle emploi et la réalisation du S2 ("délai d'initialisation ») est fixée à 15 jours ; Valorisation de parcours de formation sur d'autres : évaluation positive du «taux de projets de formation ciblés sur les métiers en tension " (c'est-àdire ceux présentant une balance excédentaire de l'offre de travail sur la demande et représentant donc un levier d'action potentiel contre le chômage).

Source : www.performance-publique.gouv.fr/farandole/2007/rap/ html/DRGPGMOPERATEURPGM103.htm

${ }^{2}$ Loi organique $n^{\circ} 2001-692 \mathrm{du} 1^{\text {er }}$ août 2001 relative aux lois de finances, allant dans le sens d'une recherche accrue de la performance budgétaire publique et reposant sur un mode de prévision des besoins et d'une transparence de l'action.
}

En effet, la connaissance du marché du travail et des éléments de définition des métiers est partiellement déléguée à des outils de positionnement; ces outils permettront de situer le profil du candidat au sein d'une échelle d'appréciation du potentiel de réussite en formation. Ces données constituent les repères objectifs à partir desquels le psychologue évaluera la concordance avec le profil du bénéficiaire, c'est-à-dire son positionnement au regard d'une norme définie (Thévenot, 2006). Nous allons voir que l'élaboration, la légitimation et la centralisation de ces ressources permettent à une logique de marché de s'incarner durant l'interaction. En effet, sont utilisés des registres qui permettent de qualifier la préconisation de juste car elle répond aux besoins exprimés par le marché de l'emploi.

Au regard de l'évolution des conditions de travail et des ressources permettant de mener leurs activités, d'artisanales, les professions relationnelles se sont progressivement managérialisées (Demailly, 2008) ; ce phénomène dénote notamment une normalisation des modes opératoires et des postures des professionnels. Dans le cas des psychologues, ce sont tout d'abord les ressources informatives qui valident ce constat. Les travaux de Naville (1972) sur les centres d'orientation de son époque nous fournissent un point de repère quant à cette évolution. Le sociologue y fait état de lacunes informatives et de discours basés majoritairement sur « des connaissances vagues issues de l'entourage, de quelques informations glanées de $c i$, de là ». Désormais, les psychologues disposent de données et supports informatifs sur les métiers, secteurs et marchés du travail. Ces savoirs sont issus d'études menées par l'INOIP ${ }^{3}$ et les Observatoires régionaux de l'emploi et de la formation. Elles s'inscrivent dans un système structuré, s'appuyant sur l'outil informatique et la participation à un réseau de construction et diffusion de connaissances organisé par le $\mathrm{SPE}^{4}$. Parmi ces supports, le psychologue dispose de référentiels qui, de leur élaboration à leurs usages, démontrent une circularité d'ordres de grandeur : ils

\footnotetext{
${ }^{3}$ Institut national de l'orientation et de l'insertion professionnelles. Les bureaux d'études de l'Afpa sont rattachés à cette structure. Ses ingénieurs de formation et d'orientation construisent et mettent à jour les contenus de formation ainsi que les tests et outils d'appui aux psychologues.

${ }^{4}$ Les fiches de poste des psychologues impliquent parfois un certain nombre de journées de participation à des études ainsi qu'à de l'activité de conseil à des organismes extérieurs au SPE.
} 
codifient un positionnement situé quant à la qualification des candidatures qu'ils permettent d'évaluer. Grâce à ces référentiels, ce positionnement se diffuse parmi les acteurs. Ils offrent ainsi à leurs utilisateurs une base commune d'analyse des situations et d'évaluation de la qualité probable des appariements entre demandeurs et formation (Eymard-Duvernay, Marchal, 2000 ; Bureau, Marchal, 2009).

Le Référentiel Emploi Activités Compétences (REAC) et le Référentiel de Certification (RC) sont des documents réalisés par les ingénieurs de l'INOIP. Issues d'un travail d'enquête auprès de représentants du marché du travail, les informations de ces référentiels sont normalisées et codifiées selon des items identiques entre tous les emplois. Le premier intervient dans le processus de création ou de réactualisation d'une formation ${ }^{5}$; il formalise ce que doit être un « emploi type ${ }^{6}$ », défini par des "activités types». L'apport en compétences nécessaires à ces activités constitue la trame du second référentiel. Celui-ci organise, d'une part, le déroulement de la formation (définition des modules et compétences à acquérir) et, d'autre part, fournit les critères d'évaluation lors de la délivrance du titre AFPA par un jury de professionnels. Ces derniers ont alors pour consigne de traduire la définition de l'employabilité comme « la capacité à se maintenir en emploi ou à se réinsérer professionnellement » (Gazier, 1990), qui questionne la mesure de l'attractivité individuelle auprès d'employeurs (Remillon, Vernet, 2009) : en tant que professionnels, recruteraient-ils ce candidat s'il se présentait pour le poste préparé par la formation? Présents en

\footnotetext{
${ }^{5}$ Après une sollicitation auprès de la DGEFP par plusieurs entreprises, une branche professionnelle, voire un organisme de formation, quant à l'opportunité de mettre en place une nouvelle formation ou bien d'en repositionner une existante, une note d'opportunité sera établie ; sa validation par la Commission professionnelle consultative relative au secteur concerné entraînera la rédaction d'un REAC.

${ }^{6}$ Un «emploi type » sera identifié par: son code ROME; sa définition ; ses conditions d'exercice et d'accès ; ses compétences rattachées; son niveau de qualification ; une description des situations particulières de mise en œuvre, des champs d'intervention, des activités-types de son noyau dur et les évolutions professionnelles. Les «activités types » détaillent ensuite: les différentes séquences qui composent l'emploi; le degré d'autonomie et de responsabilité ; les interlocuteurs et la nature des relations; les compétences requises. Les différentes compétences sont ensuite affinées selon: leur contexte de mise en œuvre; des indicateurs de performance associés à chaque activité type ; les savoirs faire et connaissances à mobiliser; les capacités relationnelles et organisationnelles nécessaires.
}

plusieurs endroits du processus de qualification, ces critères constituent des supports à la fois de conception de l'offre, de rapprochement de la demande par l'intermédiaire des psychologues et de validation de l'apprentissage par des professionnels. Leur circularité contribue à la structuration du dispositif autour des registres et informations du marché avec lesquels la codification de données établit un langage commun (Cochoy, 2000).

Par ailleurs, cette circularité qui repose sur des grilles communes de lecture et de jugement contribue à centraliser les usages des outils durant le processus. Ces usages ne sont pas prescrits au psychologue sur un mode coercitif ; ce dernier conserve sa propre rationalité, comme nous le verrons dans la seconde partie. Toutefois, une évolution quantitative des tâches, en lien avec la hausse du nombre de demandeurs de formation, engendre une nécessaire optimisation de la productivité des agents du SPE (Santelmann, 2001). La gestion coordonnée des services repose alors sur l'accessibilité et la transmission de nombreuses données recueillies par les agents de terrain durant les interactions. Aussi, chaque interaction donne lieu à des activités de codage et de mise en mémoire informatique ; cela permet d'assurer le suivi et la coordination entre les services. Or, la fonction du codage est de normaliser afin de ramener les singularités vers des modèles généraux (Thévenot, 2006) ; le principe de justice est alors appliqué selon un registre civique d'équivalence de traitement entre les bénéficiaires (Boltanski, Thévenot, 1991). S'ils soutiennent une visée d'efficacité générale du SPE, les supports de la rationalité instrumentale évoqués dans cette première partie se caractérisent aussi par une dépersonnalisation de l'accompagnement, au profit d'une logique d'action dont les bénéfices se lisent à l'échelle du marché de l'emploi. Ici se situe une ligne de tension forte dans l'activité des psychologues, lorsque son contenu, originellement fondé sur une approche relationnelle et personnalisante, se déroule dans des conditions organisationnelles propres à une « dépsychologisation » de l'accompagnement (Divay, 2008). Sans affirmer une opposition entre les visées du psychologue et celles soutenues par les outils, émerge ici une potentielle distinction entre des logiques d'action. Nous allons maintenant examiner, avec la passation des tests de niveaux, comment ces éléments organisationnels peuvent directement orienter l'interaction. 


\section{Encadré 5}

\section{Des psychologies du travail ?}

Malgré des effectifs conséquents et une certaine unité des pratiques, une faible cohésion animerait ce groupe professionnel par delà les frontières des structures. Pour Le Bianic, on assiste à un éclatement des références et des champs d'application, ainsi qu'à un code déontologique inégalement partagé et peu coercitif (2005, pp. 493-498). Les acteurs interrogés ne sont donc pas représentatifs de toute une profession, notamment au regard de l'éventail de paradigmes ayant ensuite donné lieu à de multiples postures et spécialisations: à la fin du XIXe se développent des orientations d'un côté introspectives (menant à l'approche clinique), expérimentales de l'autre (donnant naissance à la psychotechnique). L'appartenance organisationnelle primerait donc sur les spécialisations.

En 2010, nous dénombrons, en France, plus de 270 master 2, répartis en cinq grands champs (*) (santé-clinique ; développement-éducation; social-travail ; psychologie cognitive ; généraliste). Selon les orientations de la formation, les finalités d'application, les méthodes et les pratiques diffèrent. Près de 65 masters peuvent être rattachés au champ du social et du travail. Mais les compétences permettant de travailler dans le domaine de la formation ou de l'insertion peuvent aussi faire l'objet d'apprentissage dans des masters tels que " clinique de la formation " ou encore ceux relevant du champ de l'éducation intégrant des questions de formation.

Les tests et les temporalités réduites de suivi (contrairement à un suivi régulier et non limité dans le temps) positionneraient les services de I'AFPA (Association nationale pour la formation professionnelle des adultes) dans une approche psychotechnique. Toutefois, des complémentarités sont possibles entre les approches dans la mesure où les cas sont analysés en termes de particularismes, de subjectivité et d'expérience personnelle; ce que l'initiateur de la psychotechnique en France, É. Toulouse, encourageait pour que celle-ci devienne une véritable science clinique (1929). De ce point de vue, nous retrouvons cette double logique structurante des pratiques actuelles, à savoir faire valoir une écoute particulière dans un contexte de traitement de masse.

(*): Source: Guide Épicure 2010 des formations en psychologue/Association des enseignants en psychologie universitaire/ Fédération nationale des étudiants en psychologie.

\section{La passation des tests ou l'objectivation de l'adéquation offre/demande}

Dans l'évaluation de la concordance, la mesure des compétences, techniques et psychotechniques, constitue une étape incontournable. Ces tests visent à établir le potentiel de réussite des démarches de formation envisagées. Les résultats obtenus ont ainsi valeur de pronostic, construit à partir d'une rationalité instrumentale. Ce pronostic traduit, depuis la métrologie définie, une norme d'acceptabilité des niveaux de compétences. Cette norme sert de support de jugement des projets de formation (Lacourt, 2007). Elle apparaît ainsi comme un dispositif de jugement qui intègre à l'intermédiation les logiques et rationalités d'acteurs du marché et du SPE absents de la phase de recrutement.
À chaque formation va correspondre une série de tests relatifs aux compétences techniques, à des formes de logique, d'aptitudes, de perception, etc. ${ }^{7}$ Ils sont élaborés par les ingénieurs de l'INOIP qui, à partir de leurs données provenant du marché, définissent quels sont les tests adéquats selon les formations, puis fournissent un guide d'appréciation des résultats mesurés : le guide du positionnement. Cet outil se présente comme une objectivation technique de la décision finale. En effet, il équipe ses utilisateurs de repères quantitatifs prenant la forme de seuils précis d'acceptabilité. Ce contenu se prévaut d'une double légitimité : du côté des employeurs, en restituant leurs attentes quant à une « bon » recrutement; du côté de

\footnotetext{
7 Par exemple, la flexibilité cognitive, c'est-à-dire la capacité de passer d'un domaine, d'une tâche, à une autre action. Cela permet de mesurer les compétences de gestion et d'organisation du temps de travail.
} 
l'AFPA, en ciblant les capacités qui maximisent la réussite en formation. À cet effet, il repose sur une appréciation univoque des niveaux de compétences et aptitudes mesurées. Ainsi, la grille de lecture des résultats obtenus aux tests permet aux différents membres du SPE de partager une grammaire commune. En effet, la grille se présente sous la forme d'étalonnages à valeur prédictive : à des « tranches de résultats » seront associées les probabilités de réussite en formation. Ces chiffres sont construits dans une boucle rétro-évaluative à partir de données sur d'anciens stagiaires dont on met en relation les résultats aux tests, la validation du titre et le placement en emploi à l'issue de la formation : la probabilité de réussite en formation augmentera corrélativement à la maîtrise des compétences et aptitudes mesurées.

Ce travail d'objectivation fait ainsi appel à un régime de justification industrielle. Sa légitimité se fonde sur une mesure des potentiels d'apprentissage selon des calculs dont les modalités sont ajustées jusqu'à atteindre un seuil de production convenable. Par ailleurs, la mesure de l'efficacité institutionnelle du dispositif résulte du jugement prononcé par le marché lors d'embauches après la sortie de formation. En ce sens, la préconisation finale ne se co-construit pas uniquement entre psychologue et bénéficiaire; elle fait aussi intervenir des logiques liées aux rationalités d'autres acteurs. Derrière la mesure réside donc une interrogation sur l'adéquation entre un individu et le poste qu'il recherche ; la réponse à cette interrogation repose sur l'objectivation conférée par la technicité du dispositif. Le souci d'employabilité qui structure des programmes publics s'incarne ainsi dans cette étape, à partir de conventions relevant du marché du travail. L'objet et la précision de ces calculs en font un outil incontournable du S2 ; cependant, ils ont dans le même temps comme effet de limiter la justesse de la préconisation au sein des bornes définies par cet outil : une mesure en dehors des seuils fixés traduit alors un écart vis-à-vis d'une définition institutionnellement stabilisée de l'employabilité.

La présence et les effets des éléments fixes que nous avons évoqués jusque-là délimitent un cadre formalisé au dispositif S2. Ce cadre normalise des registres appliqués aux principes de justice et de justesse au cours des jugements sur les démarches de formation évaluées. La prise en compte de ces éléments normalisés, produits par des acteurs ne participant pas physiquement à l'interaction, contribuerait à réduire l'autonomie et la maîtrise de l'activité de l'utilisateur (Boussard et alii, 2010), ainsi que les particularités de sa formation. Ainsi, psychologue du travail ou clinicien, le praticien n'a pas la main sur leur usage ou leur contenu.

«On est sur des logiques de rentabilité, point barre. On n'est plus sur des logiques de progression de la personne, ou de progression sociale. Après, tout dépend peut être aussi si ça fait longtemps qu'on travaille ou pas. Moi j'ai connu autre chose, donc finalement je me rends compte maintenant et, en tant que psycho, il me semble qu'il faut qu'on prenne du recul par rapport à ça, qu'on se demande ce qu'on fait là-dedans, que sont nos limites dans ce système-là et à quoi on participe » (Psychologue SPE depuis 18 ans).

Néanmoins, le psychologue se distingue d'un agent de mesure lorsque son activité fait aussi intervenir une réflexivité issue de son expérience et de son ancienneté. En effet, la réflexivité est un produit de l'expérience ; elle s'appuie sur des situations et comparaisons fournissant aux acteurs des ordres d'interprétation des situations et produisant ainsi des routines qui orientent l'activité (Goffman, 1991). C'est alors sur la base de son expérience que le psychologue pourra potentiellement être amené à questionner le dispositif avant d'ajuster sa réponse. Ces évolutions des cadres de l'activité et l'acceptabilité des principes de contrôle sont accrues chez les plus récemment embauchés qui ne possèdent pas l'expérience empirique à vertu comparative des situations (Lavitry, 2009). Cette intériorité renvoie à la logique professionnelle des psychologues, c'est-à-dire à un système de valeurs et de représentations relevant de leur identité de professionnels avant même leur appartenance institutionnelle. La seconde partie va maintenant, d'une part, se centrer sur les implications en termes de préconisation ; d'autre part, examiner comment les espaces d'expression du psychologue sont délimités par des éléments organisationnels. 


\section{LA PRÉCONISATION FINALE : ENTRE ARBITRAGES ET NÉGOCIATIONS}

Au sein du processus de sélection de candidatures étudié, l'approche relationnelle du psychologue prend son sens dans sa lecture des logiques et intérêts qui fondent la démarche du bénéficiaire. Cette phase doit être comprise à la lumière des éléments évoqués dans la partie précédente : le psychologue dispose bien d'un pouvoir décisionnaire quant à la préconisation finale ; cependant, sa décision se construit dans un cadre délimité par les outils et supports qui objectivent des logiques et enjeux propres au marché du travail et au SPE. Lorsque concordent la volonté de l'individu, les attentes de l'institution et les besoins du marché, la validation du projet de formation n'est pas problématique. Toutefois, cette concordance n'est pas automatique. Dès lors, la co-construction de la décision consiste en un travail d'ajustement au carrefour des intérêts et enjeux en présence.

À travers trois figures d'ajustement, nous allons voir que les assemblages de logiques peuvent ramener la préconisation vers chacune des trois logiques des acteurs impliqués dans le S2. En analysant successivement comment le psychologue justifie des situations en appelant à des négociations, réajustements ou aménagements de la réponse institutionnelle, cette seconde partie mettra en lumière les espaces et ressources décisionnaires dont dispose le professionnel, mais aussi la variabilité de l'orientation de la préconisation. Au final, l'activité du professionnel se situe dans une constante tension entre les logiques en présence et les effets de contexte. C'est alors en se référant à des registres de bien commun que s'organisent les priorités de son ajustement.

\section{Négocier par les capacités}

«On ne va pas prendre forcément les meilleurs, c'està-dire que si une personne est peut-être un peu juste au niveau des tests, on sait qu'elle aura plus de difficultés mais on pense qu'elle pourra réussir. On va l'inscrire quand même, parce qu'on pense qu'elle a suffisamment de motivation ou de leviers en tout cas pouvant lui permettre de réussir » (Psychologue SPE depuis 6 ans).
Le psychologue dispose de marges de manœuvre visà-vis des règles et prescriptions organisationnelles, qui lui permettent de négocier son action selon la situation (Demazière, 1992). Ces marges de manœuvre renforcent le contrôle de sa propre activité ; elles s'incarnent ici dans la diversité des données et variables qu'il peut choisir de mobiliser et d'intégrer à la préconisation (Coutarel et alii, 2003). Certains critères de sélection possèdent ainsi un caractère indicatif et non pas éliminatoire. C'est le cas des niveaux de diplômes et prérequis pour intégrer une formation, présentés à titre de repères de connaissances et d'expérience à faire valoir pour suivre sans mal les programmes. La justesse du jugement sur ce point s'établit au sein d'une fourchette et peut faire intervenir d'autres variables que les seules capacités. Ainsi, le travail du psychologue consiste en un compromis entre données issues des tests ou du CV et éléments réflexifs produits par l'interaction. Il peut être amené à renégocier son propre avis, qu'il aurait construit uniquement d'après une lecture des éléments objectifs à sa disposition :

«Au début, elle était réticente à me faire passer les tests pour cette formation, parce le niveau d'entrée est à bac +2 et moi je n'ai même pas le bac, j'ai niveau bac. Elle avait peur que je ne sois pas prise parce que je n'ai pas le niveau ou que je n'arrive pas à suivre. Et moi je bataillais. Donc elle m'a dit: "Je vous fais passer les tests au cas où et puis on verra". Et pour le test d'électricité, elle commence à me dire que je ne l'ai pas réussi, je lui demande s'il me manque beaucoup de bonnes réponses, elle me répond "Trois", je lui fais: "C'est pas beaucoup quand même", surtout qu'avant elle m'avait dit que si je réussissais les trois tests : c'était bon; si je ne les réussissais pas du tout : je lui ai dit que c'était fini, que je laissais tomber et passais à autre chose ; et si j'étais limite : elle ferait en sorte quand même que ça passe. » (Stagiaire Afpa)

Cet affranchissement du cadre des référentiels favorise ici la logique individuelle par la renégociation de la lecture des résultats. La décision du professionnel compense en quelque sorte les limites techniques mesurées par la valorisation de la motivation personnelle et de la confiance accordée à la démarche, malgré la labilité de ces éléments de décision. De plus, ce cas présente un second argument conduisant le psychologue à négocier sa lecture des résultats : l'aspirant stagiaire évoque la possibilité de remettre en question 
son projet en cas d'échec, introduisant ainsi le risque de stopper sa dynamique personnelle, cohérente avec les postures actives que souhaite susciter le SPE. Ce psychologue déclare ainsi que : «par rapport à tel et tel élément du parcours de la personne, à sa motivation, je vais prendre le risque effectivement, je tente quand même l'inscription en formation parce que je pense qu'à un moment donné, ça peut remettre la personne sur la voie. »

L'entretien est une étape qui offre un espace de personnalisation dans le traitement de la demande, à l'inverse d'un déroulement mécanique du dispositif. Ce cas de négociation maintient la décision à un niveau minimum de cohérence avec les prérequis institutionnellement définis. Le « risque » soulevé par le psychologue repose sur l'approche relationnelle. En s'y référant ainsi, le jugement bascule d'une recherche d'efficacité des appariements vers une logique que l'on pourrait qualifier de service rendu à l'usager. En effet, en valorisant l'argumentaire du bénéficiaire face à une évaluation technique qui déprécie le potentiel de réussite du projet de formation, le psychologue fait primer les aspirations de ce dernier sur les éléments formels à sa disposition. Toutefois, ce type d'ajustement à la marge peut se révéler insuffisant lors de situations impliquant non pas de négocier mais d'arbitrer entre des logiques.

\section{Des logiques contradictoires}

La volonté institutionnelle de privilégier des postures actives peut faire émerger des contradictions entre logique institutionnelle et situation du bénéficiaire. Le second cas exposé témoigne d'écarts potentiels de conceptions entre membres du SPE quant à la finalité du S2. Le bénéficiaire est adressé par un conseiller Pôle emploi qui considère la solution formation comme une réponse pertinente à la problématique individuelle. Un projet est donc initié selon le processus, qu'il convient ensuite de valider ou non. Dans le cadre d'une gestion parfaitement rationnelle de la coordination des services, issue des principes du nouveau management public, l'opérateur n'aurait pas à revenir sur le travail effectué en amont : cette division institutionnelle du travail définit les apports singuliers selon les fonctions propres à chaque composante du SPE. Or, cette modalité d'émergence d'un projet de formation révèle une facette particulière du processus. En effet, l'organisation prévoit, dans le même temps, un espace d'expression du jugement au cours du processus, renvoyant à ce que Maggi repère comme des zones de discrétion (1996 ; 1999). Celles-ci permettent au psychologue de réajuster la conception de la juste préconisation institutionnelle. Le psychologue trouve alors dans cette marge de manœuvre un renforcement de sa capacité à définir son rôle professionnel :

«Un cas récent: une femme qui vient parce que l'ANPE lui demande de faire une formation en montage câblage. Assez rapidement, je m'aperçois que la femme a 53 ans, le permis mais pas de voiture, qu'elle élève un jeune de 18 ans qui est en BEP et qu'elle a 500 euros par mois pour vivre. Et elle est envoyée par l'ANPE pour faire un stage qui dure quasiment 10 mois, qui va lui demander de très gros efforts au niveau scolaire, etc. Et quand je lui explique un peu le contenu de la formation : le calcul, la lecture de plans, de cartes, la femme me dit "Mais attendez, je suis incapable de faire ça, psychologiquement, intellectuellement. Moi ce que je veux, c'est vite travailler".

$\mathrm{Q}$ : « Cette personne avait des notions en montage câblage?»

$\mathrm{R}$ : «Non, du tout. Mais il y a des besoins en emploi dans ce secteur près d'ici » (Psychologue SPE depuis 18 ans).

La zone de discrétion révèle un décalage entre des injonctions à la mise en action des bénéficiaires et une logique adéquationniste visant une correspondance des compétences à construire du côté de la demande (Garraud, 1995) : on propose au bénéficiaire des actions orientées en priorité vers des secteurs ciblés en termes d'emploi, rendant secondaire la prise en compte de sa situation personnelle et de son profil. Ce type de préconisation se construit en priorité à partir des besoins du marché du travail auxquels on cherche à faire correspondre les besoins individuels. Dès lors, la nature du poste visé et son adéquation avec le profil et les problématiques personnelles apparaissent comme dépersonnalisées dans ce type d'adressage pour un S2. L'employabilité visée par le dispositif apparaît ici comme une transaction entre individu et marché, demandant au premier de faire correspondre ses attentes à celles du second. 
Lorsqu'il requalifie l'orientation du bénéficiaire par le SPE, jugeant trop élevé l'écart entre le profil individuel et la prescription institutionnelle, le psychologue fonde sa préconisation sur des registres propres à sa logique professionnelle (approche qualitative d'un registre civique) et non relatifs à une logique quantitative d'un registre industriel (Foucauld, Thévenot, 1995). Les marges de manœuvre du psychologue octroyées par la zone de discrétion apparaissent alors centrales dans l'affirmation de son genre professionnel. En effet, le genre professionnel correspond à un système tacite d'évaluation des situations de travail : dans ses travaux, Yves Clot le définit comme un corps intermédiaire symbolique organisant le rapport à l'activité et facteur de cohésion parmi les professionnels qui en partagent les contenus (1999). Son affirmation engage alors l'identité professionnelle telle que l'opérateur la conçoit. La situation contradictoire représente donc, chez l'opérateur, une menace pour ce genre. Co-construire une préconisation revient à lier les enjeux du bénéficiaire à ceux du psychologue.

Cependant, il serait réducteur de ne considérer la logique professionnelle des psychologues que comme un support des aspirations des bénéficiaires. Un dernier type de cas de contingences externes à la situation individuelle rend compte d'une orientation de l'arbitrage autour des enjeux de l'institution.

\section{Logique d'institution}

Les organismes publics sont évalués sur leur efficience, rapportant les résultats obtenus sur les moyens déployés. Aussi, l'optimisation des effectifs constitue une préoccupation institutionnelle forte. La phase de sélection des candidatures occupe une place centrale et certains de ses enjeux échappent au psychologue, tout en relevant de sa responsabilité aux yeux de l'organisation.

Une nouvelle contradiction peut s'immiscer dans la construction de la préconisation, cette fois-ci entre logiques de marché et d'institution, partant du principe que la volonté du bénéficiaire est respectée. Le statut non décisif des outils se révèle un levier d'action fort utile lorsque, à l'approche d'un début de formation, les effectifs ne sont pas optimisés. Les seuils d'acceptabilité des compétences requises sont alors parfois revus à la baisse, assouplissant les critères de sélection. Cette pratique, axée sur la situation de l'organisme de formation, sert une logique gestionnaire d'institution; cette logique prévaut sur la logique économique (les niveaux de capacités et de compétences initiaux diminuent et donc avec eux les critères formalisés de l'employabilité), mais aussi sur la logique individuelle (le pari évoqué plus haut s'établit sur une incertitude plus forte, le risque d'échec se reporte sur le destinataire). Avouée du bout des lèvres par certains psychologues, son ampleur reste difficile à évaluer :

« Cela dit, ça m'est arrivé, si je sens que la personne, vraiment, c'est ça qu'elle veut. Le problème, c'est que quand on leur dit "Là, ça va être trop difficile pour vous, je suis pas d'accord pour que vous y alliez parce que c'est pas une solution viable", la personne elle répond "Mais moi je suis motivé pour le faire, je vous garantis que je vais réussir" parce qu'elle ne perçoit pas les difficultés dans lesquelles elle va être en fait. Ça c'est difficile... Donc quelquefois et bien, j'avoue que je laisse faire parce que si après tout la personne veut y aller, s'il y a de la place, ça prend la place à personne, il y a par exemple 12 places et il en reste 4 de libres. Ça m'est arrivé d'accepter ça, c'est rare, mais ça m'est arrivé. » (Psychologue SPE depuis 15 ans)

En outre, ce dernier cas rend compte de la structuration de l'activité par des logiques d'efficacité publique. Si les plus récentes recrues parviendraient à s'en accommoder, faute d'expériences comparatives, l'expérience semble éclairer comment la pluralité de logiques en présence peut être source de tensions dans l'activité :

« Je trouve qu'on a moins de marges de manouvre personnellement. On essaie de rester en accord avec le travail qu'on fait, on s'en accommode, mais ce n'est pas sans un certain stress au travail aujourd'hui. On est dans des injonctions paradoxales en fait. » (Psychologue SPE depuis 8 ans)

La multiplication des contraintes et cadrages organisationnels visant une efficacité institutionnelle et de marché rend nécessaires des ajustements, voire des arbitrages, entre les logiques en présence. C'est alors vis-à-vis de sa propre logique professionnelle que le psychologue pourra se trouver en situation de tension 
quand, à la différence du cas précédent, la situation le conduit à faire primer un enjeu organisationnel, voyant le bien commun convoqué glisser depuis une justification relationnelle vers un registre industriel d'optimisation des postes de formation ${ }^{8}$. Ce dernier cas révèle que si les marges de manœuvre présentes dans le processus constituent des ressources pour un ajustement des préconisations, l'orientation de cet ajustement peut se référer à des niveaux d'action différents auxquels peut se rattacher le psychologue : local (individu); organisationnel (SPE); général (marché de l'emploi).

Au final, l'activité du psychologue met avant tout en relief la recherche d'un compromis entre les logiques plus que l'accompagnement unique du bénéficiaire. Ceci marque une évolution potentielle dans la définition que le professionnel se fait de son activité ; cette évolution peut être source de tensions selon qu'il se reconnaît ou pas dans les logiques mises en œuvre. Par les cas présentés, la notion de co-construction témoigne moins d'une élaboration commune entre institution et bénéficiaire que d'un compromis entre différents niveaux de contingences, dont une large part échappe au bénéficiaire.

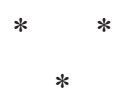

Dans le cadre du nouveau management public et de la réorganisation du service public de l'emploi autour d'une rationalisation des activités, l'épreuve de jugement que constitue le S2 (rappelons que le S2 consiste en une prestation de conseil et d'accompagnement dont l'objet est de co-construire et de valider avec le bénéficiaire un projet de formation), et plus généralement les dispositifs d'intermédiation publique entre offre et demande de travail reposent de façon croissante sur une objectivité outillée et une coordination entre acteurs. Présenté comme recherchant la «solution la plus adaptée à la personne », le dispositif apparaît comme un processus de co-construction dont l'issue la préconisation - intègre des éléments et enjeux relatifs au bénéficiaire, mais aussi à l'organisation, au marché du travail et à la position professionnelle du

\footnotetext{
${ }^{8}$ Cela renvoie à la difficulté qu'éprouvent les psychologues à caractériser l'apport général de leur fonction au sein du SPE (d'où la référence au «bien commun »).
}

psychologue. Les situations de correspondance ou d'écart minime entre profil du bénéficiaire et attentes formalisées du SPE n'induisent pas une véritable co-construction mais la validation d'une convergence d'intérêts entre les acteurs concernés. Le cas échéant, évoquer une co-construction des projets par le SPE renvoie alors à un travail d'ajustement entre producteurs du système de formation, opérateurs de l'action publique et bénéficiaires des programmes d'accompagnement. Cet ajustement vise à trouver une solution qui réponde à des enjeux de multiples niveaux, se rapportant tout autant à la lecture de la situation d'interaction qu'à la situation du bénéficiaire.

L'interaction avec le psychologue apparaît alors comme l'étape permettant au jugement de basculer de registre et de revaloriser certaines logiques jusquelà peu représentées. L'accompagnement public ne peut ainsi être dissocié de la subjectivité de ceux qui le mettent en œuvre. Dès lors, c'est principalement dans les marges de manœuvre et zones de discrétion ménagées par l'organisation que cette subjectivité peut s'exprimer. Lorsque la situation lui semble le nécessiter, c'est au sein de ces espaces que les modalités de la décision finale seront requalifiées par le psychologue.

L'analyse fait alors émerger un second niveau d'enjeu concernant la capacité du professionnel à définir sa propre activité. En effet, les éléments organisationnels participent de l'orientation de l'action, fixent une part du cadre des activités du psychologue et formalisent la façon de les mener. Leur présence dans l'interaction soutient une coordination entre les différents participants du réseau d'action que constitue le SPE. Ils médiatisent ainsi des conceptions externes au psychologue de ce qu'est une juste sélection en formation. Ce cadre insère ainsi le plan local de l'interaction dans un niveau d'action et d'enjeu plus global. Et nous touchons là à l'une des tensions que la position du psychologue infère à son action : voyant sa logique professionnelle définir des enjeux qui parfois divergent de ceux promus par son environnement organisationnel, la définition qu'il se donne de son rôle en fonction de son genre professionnel (Y. Clot, 1999) peut tendre vers une désynchronisation des enjeux. La présence de marges de manœuvre permet de régler des situations de possible schizophrénie professionnelle (Dubois, 2001), lorsque le psychologue est confronté 
à plusieurs définitions de son rôle et, attenantes, plusieurs issues possibles des interactions.

Enfin, le recours à la notion de genre professionnel souligne la part de l'expérience empirique au sein de la logique professionnelle. Elle fournit notamment aux psychologues des registres d'interprétation et de comparaison des situations de travail. Les renouvellements générationnels des effectifs interrogent alors sur les évolutions potentielles de cette logique professionnelle que nous avons étudiée. En effet, la perception des missions fondant l'activité distingue, dans certaines catégories professionnelles, des sousgroupes selon qu'ils ont ou non expérimenté un changement de statut: dans le cas des conseillers Pôle emploi par exemple, la dimension de contrôle des bénéficiaires serait plus facilement acceptée par les recrues les plus récentes, qui n'ont pas connu de modification de leur statut (Lavitry, 2008). Le récent rattachement des psychologues du travail de l'AFPA au Pôle emploi pose alors, à terme, la question de l'évolution de leur logique professionnelle, à la croisée des autres logiques d'action présentes dans leur activité.

\section{Bibliographie}

Beraud M., Eydoux A. (2009), «Activation des chômeurs et modernisation du service public de l'emploi : les inflexions du régime français d'activation », Travail et Emploi, $\mathrm{n}^{\circ} 119$.

Bessy C., Eymard-Duvernay F., Gomel B., Simonin B. (1995), «Les politiques publiques de l'emploi : le rôle des agents locaux », Cahiers du centre d'études de l'emploi, $\mathrm{n}^{\circ} 34$, Paris, PUF, pp. 3-34

Boltanski L., Thévenot L. (1991), De la justification. Les économies de la grandeur, Paris, Gallimard.

Boussard V. (2001), « Quand des règles s'incarnent, l'exemple des indicateurs prégnants », Sociologie $d u$ travail, vol. 43 (4), pp. 533-551.

Boussard V., Demazière D., Milburn P. (dir.), (2010), L'injonction au professionnalisme. Analyses d'une dynamique plurielle, Rennes, PUR.

Boussard V., Maugeri, S. (dir.), (2003), Du politique dans les organisations. Sociologies des dispositifs de gestion, Paris, L'Harmattan.

Bureau M.-C., Marchal E. (2009), «Incertitudes et médiations au cœur du marché du travail », Revue française de sociologie, vol. 50 (3), pp. 573-598.
Clot Y. (1999), La fonction psychologique du travail, Paris, PUF, coll. « Le travail humain ».

Cochoy F. (2000), « De 1"“AFNOR" à "NF", ou la progressive marchandisation de la normalisation industrielle », Réseaux, n 102, pp. 63-89.

Coutarel F., Daniellou F., Dugue B. (2003), « Interroger l'organisation du travail au regard des marges de manœuvre en conception et en fonctionnement », Pistes, vol. 5 (2), 24 p.

Demailly L. (2008), Politiques de la relation. Approche sociologique des métiers et activités professionnelles relationnelles, Villeneuve d'Ascq, Presses Universitaires du Septentrion.

Demazière D. (1992), « La négociation des identités des chômeurs de longue durée ", Revue française de sociologie, vol. 33 (3), pp. 35-363.

Dubar C. (1989), «Vingt ans de formation continue en France: 1'utopie de la "2 2 chance" aux réalités contrastées de la gestion de l'emploi », Éducation permanente, $\mathrm{n}^{\circ}$ 98, pp. 163-171.

Dubar C., Gadéa C. (1998), «Évolution de la promotion sociale et dynamique des formes identitaires », Éducation Permanente, $\mathrm{n}^{\circ} 136$ (3), p. 81. 
Dubois V. (2001), Action publique et processus d'institutionnalisation. Sociologie des politiques culturelle et linguistique et du traitement bureaucratique de la misère, Habilitation à diriger les recherches en sociologie de l’Université Paris I.

Divay S. (2008), « Psychologisation et dépsychologisation de l'accompagnement des chômeurs ", Sociologies pratiques, $\mathrm{n}^{\circ} 17$, pp. 55-66.

Eymard-Duvernay F. (1989), «Conventions de qualité et formes de coordination », Revue économique, vol. 40 (2), pp. 329-360.

Eymard-Duvernay F. (2008), «Justesse et justice dans les recrutements », Formation Emploi, n 101 , pp. 55-69.

Eymard-Duvernay F., Marchal E. (2000), «Qui calcule trop finit par déraisonner: les experts du marché du travail », Sociologie du travail, vol. 42 (3), pp. 411-432.

Foucauld J.-B. de, Thévenot L. (1995), « Évolution des politiques sociales et transformation de l'action publique », Cahiers du Centre d'Études de l'Emploi, n 34, pp. 319-349.

Garraud P. (1995), « La mise en œuvre des politiques de lutte contre le chômage par la formation: les contraintes du service public de l'emploi en France ", Sociologie du travail, vol. 37 (4), pp. 675-696.

Gazier B. (1990), «L'employabilité : brève radiographie d'un concept en mutation », Sociologie $d u$ travail, vol. 32 (4), pp. 575-584.

Gazier B. (2009), « Parcours professionnels et conditions de travail : dimensions individuelles et collectives de l'employabilité "), Éducation permanente, $\mathrm{n}^{\circ} 181$, pp. 149-153.

Goffman E. (1991), Les cadres de l'expérience, Paris, Minuit.

Lacourt I. (2007), «Des catégories de l'action publique à l'épreuve de la subjectivité », Droit et société, vol. 46, pp. 219-230.
Lagroye J. (2002), Sociologie politique, Paris, Dalloz, Presses de Sciences Po.

Lavitry L. (2008), Les conseillers à l'emploi: de la construction d'une profession à des figures de conseillers, Séminaire du LEST, Aix en Provence, 3/11/08 (consultable sur H.A.L.).

Lavitry L. (2009), « De l'aide au contrôle? Les conseillers à l'emploi à l'épreuve de la personnalisation », Travail et Emploi, n 19, pp. 63-75.

Le Bianic T. (2005), Les "ingénieurs des âmes». Savoirs académiques et pratiques des psychologues du travail de l'entre deux guerres à nos jours, Thèse de l'Université Aix-Marseille 2.

Le Bianic T., Rot G. (2009), «Cadrer les cadres » in Vatin F. (dir.), Évaluer et valoriser. Une sociologie économique de la mesure, Toulouse, PUM, pp. 155-174.

Loubat J.-R. (2000), « Prestataires et bénéficiaires doivent passer un contrat», Lien Social, $\mathrm{n}^{\circ} 523$, pp. 4-8.

Maggi B. (1996), «La régulation du processus d'action de travail» in Cazamian P., Hubault F., Noulin M. (dir.), Traité d'ergonomie, Toulouse, Octarès, pp. 637-662.

Maggi B., Massino G. (1999), « Niveaux de décisions et modes de régulation : l'autonomie et la discrétion dans le processus de travail», Actes du Séminaire Contradictions et dynamique des organisations, Paris.

Mériaux O. (2009), «Les parcours professionnels : définition, cadre et perspectives ", Éducation permanente, $\mathrm{n}^{\circ} 181$, pp. 11-22.

Merle V. (2009), « La formation des adultes en France: une politique publique en devenir» in Barbier J.-M., Bourgeois É., Chapelle G., RuanoBorbalan J.-C. (dir.), Encyclopédie de la formation, Paris, PUF. 
Naville P. (1972), Théorie de l'orientation professionnelle, Paris, Gallimard.

Nizet J. (2009), « Les évolutions idéologiques dans le domaine de la formation », in Barbier J.-M., Bourgeois É., Chapelle G., Ruano-Borbalan J.-C. (dir.), Encyclopédie de la formation, Paris, PUF, pp. 967-996.

Pattaroni L. (2007), « Le sujet en l'individu. La promesse d'autonomie du travail social au risque d'une colonisation par le proche » Droit et société, vol. 46, pp. 203-218.

Remillon D., Vernet A. (2009), « De l'inaptitude à l'employabilité » in Vatin F. (dir.), Évaluer et valoriser. Une sociologie économique de la mesure, Toulouse, PUM, pp. 115-137.
Santelmann P. (2001), La formation professionnelle, nouveau droit de l'homme?, Paris, Gallimard.

Thévenot L. (1983), «L'économie du codage social », Critiques de l'Économie Politique, n 3-24, pp. $188-222$.

Thévenot L. (2006), L'action au pluriel, Paris, La Découverte.

Toulouse É. (1929), «Les relations actuelles de la psychotechnique et de la psychiatrie », $4^{e}$ Conférence internationale de psychotechnique (1927), Paris, Alcan, pp. 533-541.

Weller J.-M. (1999), L'État au guichet, Paris, Desclée de Brouwer.

\section{Résumé}

\section{L'intermédiation à la croisée des logiques d'acteurs : les psychologues du travail}

\section{Benjamin Saccomanno}

Acteur de la mise en relation entre offre et demande de travail, le service public de l'emploi propose des formations qualifiantes afin d'accroître l'employabilité de ses publics. Cet article s'intéresse à l'étape de sélection pour ces formations. II interroge la façon dont l'institution objective son appréciation de ce qui constituera un "bon » projet de formation. À cet effet, outils, supports informatifs et dispositifs de mesure encadrent et impactent l'activité des psychologues chargés du recrutement. Au final, leur travail consiste à la fois à assembler des logiques parfois divergentes, voire contradictoires, tout en tentant de maintenir leurs propres marges de manœuvre de professionnels. La co-construction des projets de formation, avec les bénéficiaires, apparaît alors comme l'enjeu de ces logiques, dont la coexistence est source de tensions pour le psychologue.

Mots clés

Service public de l'emploi, chômeur, psychologue, projet de formation, projet professionnel, métier de l'emploi

Journal of Economic Literature : J 24, M 53 